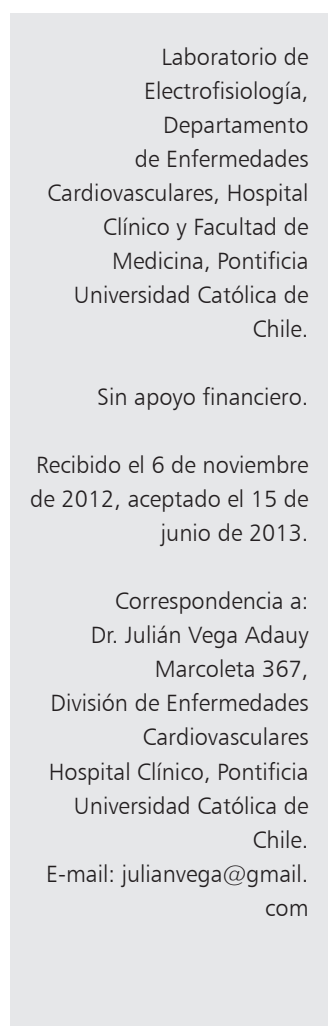

\section{Tormenta eléctrica en síndrome de Brugada tratada exitosamente con infusión de isoproterenol}

\author{
JULIÁN VEGA, ANDRÉS ENRÍQUEZ, ALEX BITTNER, \\ ISMAEL VERGARA, PATRICIA FRANGINI, MARIANA BAEZA, \\ ISLANDIA MILLAPÁN, ROLANDO GONZÁLEZ
}

\section{Electrical storm in Brugada syndrome successfully treated with isoproterenol. Report of a case}

\begin{abstract}
We report a 22-year-old male who experienced several episodes of syncope within a timeframe of few hours. In the emergency room, multiple ventricular fibrillation episodes where documented along with a type 1 Brugada ECG pattern. Isoproterenol in continuous infusion was started, normalizing the ECG and avoiding further arrhythmia recurrences. The patient was implanted with an automated defibrillator and discharged 3 days after admission.
\end{abstract}

(Rev Med Chile 2013; 141: 1340-1343).

Key words: Brugada Syndrome; Isoproterenol; Syncope; Ventricular Fibrillation.
E 1 síndrome de Brugada es un trastorno eléctrico primario del corazón caracterizado por bloqueo incompleto de rama derecha, supradesnivel del segmento ST en precordiales derechas $(\mathrm{V} 1-\mathrm{V} 3)$ y predisposición a muerte súbita por fibrilación ventricular ${ }^{1}$. Es de transmisión autosómica dominante y se debe a mutaciones "pérdida de función" en genes que codifican la subunidad alfa del canal de sodio $(\mathrm{Na})$ dependiente de voltaje SCN5A ${ }^{2}$. Tiene una mayor prevalencia en hombres y en general su presentación clínica ocurre antes de los 40 años. Su prevalencia se estima en 5 por 10.000 habitantes y se atribuye como responsable de hasta $20 \%$ de las muertes súbitas en ausencia de cardiopatía estructural ${ }^{3}$.

Normalmente el potencial de acción del epicardio exhibe una fase 1 más prominente que el del endocardio (forma de raíz cuadrada) debido a preponderancia local de la corriente Ito. En el síndrome de Brugada una reducción en la corriente de Na debida a mutación del SCN5A produce un predominio de las corrientes de eflujo de cationes (Ito) sobre las corrientes de influjo (INa e ICaL).
Esto lleva a la pérdida del domo de dicho potencial de acción en las células epicárdicas y es responsable del patrón electrocardiográfico característico de Brugada, con onda J y supradesnivel del ST en precordiales derechas debido a un importante gradiente de voltaje transmural. La heterogeneidad en la refractariedad de las distintas capas favorecería los mecanismos de reentrada ${ }^{4}$ y la presencia de arritmias ventriculares polimorfas.

Reportamos el caso de un paciente con síndrome de Brugada que debutó por un cuadro de tormenta eléctrica y fue tratado exitosamente con infusión de isoproterenol.

\section{Caso clínico}

Paciente masculino de 22 años de edad consultó al servicio de urgencia de nuestra institución por síncope recurrente. Estando en reposo en su casa, una hora posterior a haber finalizado entrenamiento de boxeo, presentó compromiso súbito de conciencia de aproximadamente un minuto de 
Síndrome de Brugada e isoproterenol - J. Vega et al

duración, con recuperación completa posterior. En las dos horas siguientes repitió episodios sincopales de similares características en tres oportunidades. En el hall de acceso al hospital presentó nuevo cuadro sincopal, por lo que fue trasladado al box de urgencia para evaluación inmediata. $\mathrm{Al}$ ingreso se constató presión arterial de 126/82 $\mathrm{mmHg}$, frecuencia cardiaca de $82 \mathrm{lpm}$, saturación de $99 \%$ y temperatura axilar de $36^{\circ} \mathrm{C}$. El paciente no refería comorbilidades significativas ni antecedentes familiares de cardiopatía. Durante realización del ECG inicial paciente repitió síncope, objetivándose en el trazado fibrilación ventricular (Figura 1). Se iniciaron maniobras de reanimación y el paciente convirtió a ritmo sinusal después de unos segundos. Se repitió un ECG, que mostraba imagen de bloqueo incompleto de rama derecha, supradesnivel del ST e inversión de la onda T en precordiales derechas, sugerente de patrón Brugada tipo 1 (Figura 2). A los pocos minutos se evidenció nuevo episodio de fibrilación ventricular y se procedió a la inmediata desfibrilación con 200 Joules de corriente bifásica, dando salida a ritmo sinusal. El paciente fue hospitalizado en Unidad Coronaria, donde fue sedado con lorazepam y se decidió iniciar infusión de isoproterenol a dosis de $0,25 \mathrm{ug} / \mathrm{min}$. Se registra ECG durante infusión de isoproterenol, en el cual se apreció regresión del patrón de Brugada dado por normalización del segmento ST en V1-V2, desaparición de la imagen de bloqueo de rama derecha y restauración de la polaridad de la onda T (Figura 3). Ecocardiograma descartó patología estructural y exámenes de laboratorio no mostraron alteraciones significativas del medio interno. Evolucionó favorablemente, suspendiendo infusión antiarrítmica al segundo día e implantando cardiodesfibrilador bicameral (Protecta CRT-D, Medtronic) sin incidentes. Fue dado de alta en buenas condiciones al tercer día de hospitalización sin medicación antiarrítmica. En control ambulatorio al mes no se registraron eventos arrítmicos en chequeo del dispositivo.
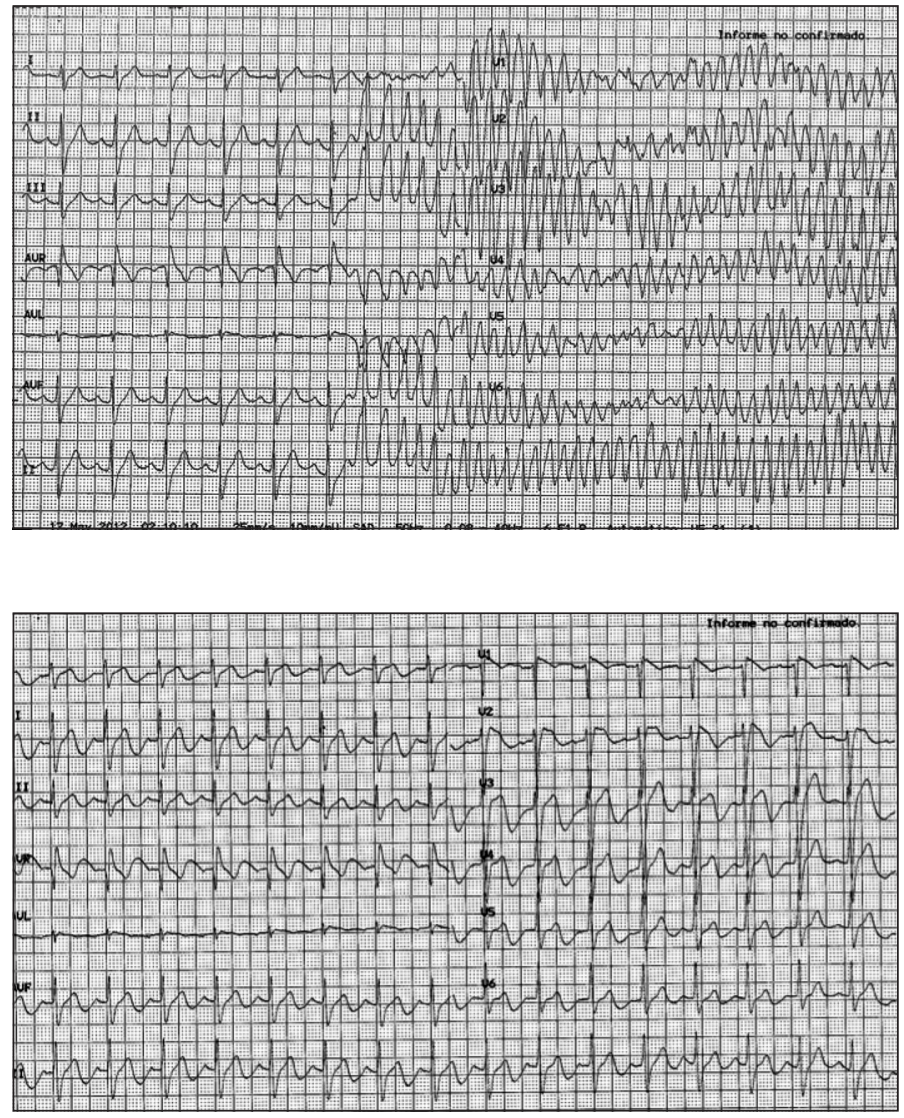

Figura 1. ECG de ingreso. Se observa momento exacto de inicio de la fibrilación ventricular.

Figura 2. ECG post conversión espontánea a ritmo sinusal. Se observa el patrón Brugada tipo 1 característico con elevación del segmento ST y patrón de bloqueo de rama derecha en derivaciones V1 y V2. 


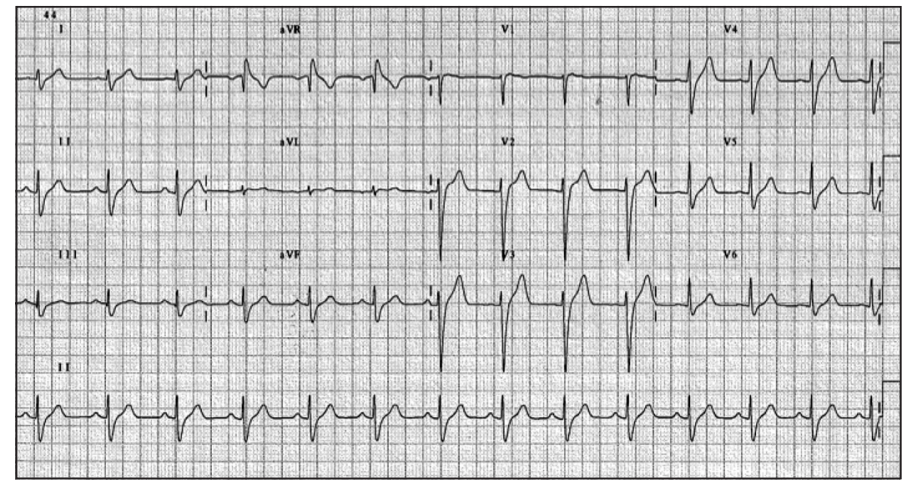

Figura 3. ECG durante infusión de Isoproterenol. ECG tomado 15 min post inicio de infusión de isoproterenol. Se observa normalización del patrón Brugada, con regresión de supradesnivel del ST en precordiales derechas.

\section{Discusión}

Se define tormenta eléctrica como la aparición de 3 o más episodios de taquicardia ventricular sostenida o fibrilación ventricular durante un período de $24 \mathrm{~h}$. Su presentación en el contexto de síndrome de Brugada es un fenómeno raro ${ }^{5}$, pero potencialmente fatal y puede ser la primera manifestación de la enfermedad ${ }^{6}$.

El manejo es complejo y se sustenta mayormente en reportes de casos aislados. La terapia farmacológica tiene como objetivo restaurar el balance entre las corrientes iónicas que tienen lugar durante la fase inicial del potencial de acción epicárdico. El agente más utilizado en los reportes publicados ha sido el agonista beta-adrenérgico isoproterenol, que es capaz de normalizar el patrón electrocardiográfico y prevenir la inducción de fibrilación ventricular en el estudio electrofisiológico ${ }^{4,7-9}$. Se postula que la estimulación betaadrenérgica aumenta la corriente ICaL y restaura el domo de los potenciales de acción epicárdicos, reduciendo la heterogeneidad transmural. Esto normaliza el desnivel del ST y previene la fibrilación ventricular. En el presente caso el fármaco tuvo un efecto dramático, con completa normalización del ECG poco después de su inicio y cese de las arritmias ventriculares. Beneficios similares han sido reportados con el uso de orciprenalina, otro agonista beta-adrenérgico ${ }^{10}$.

Si bien el isoproterenol es el tratamiento agudo de elección para suprimir la tormenta eléctrica, su suspensión puede llevar a recurrencia de la fibrilación ventricular. En estos casos habitualmente es necesario el inicio de medicamentos orales con el fin de disminuir y discontinuar la infusión de isoproterenol ${ }^{9}$. Dentro de los agentes orales el más usado ha sido la quinidina, un antiarrítmico clase IA que bloquea los canales Ito en el epicardio del ventrículo derecho, lo que permite restaurar el domo del potencial de acción epicárdico, reduciendo el gradiente de voltaje transmural durante la fase plateau y normalizando el desnivel del $\mathrm{ST}^{10-12}$. Este fármaco, sin embargo, no se encuentra disponible en nuestro país. Otra alternativa de terapia oral incluye al cilostazol, la denopamina y el bepridil. El cilostazol es un inhibidor de la fosfodiesterasa tipo III que aumenta ICaL vía aumento del AMPc intracelular ${ }^{13}$. La denopamina es un estimulante adrenérgico que actúa probablemente aumentando ICaL y el bepridil actúa por bloqueo de Ito $^{9}$. Finalmente, es importante recordar que para la prevención de muerte súbita en el síndrome de Brugada no existe beneficio demostrado de la terapia antiarrítmica y la única medida con impacto real en la sobrevida es el implante de un cardiodesfibrilador.

\section{Referencias}

1. Brugada P, Brugada J. Right bundle branch block, persistent ST-segment elevation and sudden cardiac death. J Am Coll Cardiol 1992; 20: 1391-6.

2. Antzelevitch C. The Brugada syndrome: ionic basis and arrhythmia mechanisms. J Cardiovasc Electrophysiol 2001; 12: 268-72.

3. Antzelevitch C, Brugada P, Borggrefe M, Brugada J, Brugada R, Corrado D, et al. Brugada syndrome: Report of the Second Consensus Conference: Endorsed by the Heart Rhythm Society and the European Heart Rhythm Association. Circulation 2005; 111: 659-70.

4. Maury P, Couderc P, Delay M, Boveda S, Brugada J. Electrical storm in Brugada syndrome successfully treated using isoprenaline. Europace 2004; 6: 130-3. 
5 Chalvidan T, Deharo JC, Dieuzaide P, Defaye P, Djiane P. Near fatal electrical storm in a patient equipped with an implantable car- dioverter defibrillator for Brugada syndrome. Pacing Clin Electro- physiol 2000; 23: 410-2.

6. Dinckal MH, Davutoglu V, Akdemir I, Soydinc S, Kirilmaz A, Aksoy M. Incessant monomorphic ventricular tachycardia during febrile illness in a patient with Brugada syndrome: fatal electrical storm. Europace: European pacing, arrhythmias, and cardiac electrophysiology: journal of the working groups on cardiac pacing, arrhythmias, and cardiac cellular electrophysiology of the European Society of Cardiology 2003; 5: 257-61.

7. Maury P, Hocini M, Haissaguerre M. Electrical storms in Brugada syndrome: review of pharmacologic and ablative ther- apeutic options. Indian Pacing Electrophysiol J 2005; 5: 25-34.

8. Jongman JK, Jepkes-Bruin N, Ramdat Misier AR, Beukema WP, Delnoy PPHM, Oude Luttikhuis H, et al. Electrical storms in Brugada syndrome successfully treated with isoproterenol infusion and quinidine orally. Neth
Heart J 2007; 15: 151-4.

9. Ohgo T, Okamura H, Noda T, Satomi K, Suyama K, Kurita $\mathrm{T}$, et al. Acute and chronic management in patients with Brugada syndrome associated with electrical storm of ventricular fibrillation. Heart Rhythm 2007; 4: 701-2.

10. Kyriazis K, Bahlmann E, van der Schalk H, Kuck K. Electrical storm in Brugada syndrome successfully treated with orciprenaline; effect of low-dose quinidine on the electrocardiogram. Europace 2009; 11 (5): 665-6.

11. Belhassen B, Glick A, Viskin S. Efficacy of quinidine in high-risk patients with Brugada syndrome. Circulation 2004; 110: 1731-7.

12. Alings M, Dekker L, Sadee A, Wilde A. Quinidine induced electrocardiographic normalization in two patients with Brugada syndrome. Pacing Clin Electrophysiol 2001; 24: 1420-2.

13. Tsuchiya T, Ashikaga K, Honda T, Arita M. Prevention of ventricular fibrillation by cilostazol, an oral phosphodiesterase inhibitor, in a patient with Brugada syndrome. J Cardiovasc Electrophysiol 2002; 13: 698-701. 